\title{
Small Antennas: Miniaturization Techniques and Applications 2016
}

\author{
Wenhua Yu, ${ }^{1}$ Yingsong $\mathrm{Li}^{2}{ }^{2}$ and Manos M. Tentzeris ${ }^{3}$ \\ ${ }^{1} 2$ COMU Inc., Fairfax, VA, USA \\ ${ }^{2}$ College of Information and Communications Engineering, Harbin Engineering University, Harbin 150001, China \\ ${ }^{3}$ School of Electrical and Computer Engineering, Georgia Institute of Technology, Atlanta, GA 30308, USA
}

Correspondence should be addressed to Wenhua Yu; wenyu@2comu.com

Received 1 September 2016; Accepted 1 September 2016

Copyright (c) 2016 Wenhua Yu et al. This is an open access article distributed under the Creative Commons Attribution License, which permits unrestricted use, distribution, and reproduction in any medium, provided the original work is properly cited.

The development of small antennas for modern communications, for both portable terminals and radar purposes, has been widely studied over many years. In recent decades, this topic is experiencing an ever increasing concentration, thanks to the antenna miniaturization techniques and printed antenna techniques. Recently reported antenna techniques pave the way to new added-value designs of small antennas for mobile devices and military applications and even for healthcare detections and enable full exploitation for the integration of the antenna and radio frequency front-ends. However, the antennas used in modern communication equipment have unique challenges in design theory and in implementation, which need new development in the antenna design and geometry design in small antenna creation.

The papers included in this special issue provide a wide view of small antenna designs, which includes the antenna designs and applications in communication system. This special issue comprises the antenna designs for long-term evolution (LTE), radio-frequency identification (RFID), global navigation satellite system (GNSS), and mobile devices. Additionally, the optimal electric dipole antenna model and its application have also been included in this special issue.

As for the LTE antenna design, the authors created a spiral slotted microstrip antenna to meet the requirements of $700 \mathrm{MHz}$ band application, which can also be used for terrestrial digital television. The antenna has been well designed and measured for obtaining good impedance and gain. For mobile communications, a quad-band slot antenna and a dual-polarization antenna have been reported and investigated in detail. The quad-band slot antenna has been presented for personal communications service (PCS), universal mobile telecommunications system (UMTS), wideband code-division multiple access (WCDMA), Bluetooth, wireless local area networks (WLAN), and worldwide interoperability for microwave access (WiMAX) applications and the dual-polarization antenna has been developed for $1801 \mathrm{MHz}-1827 \mathrm{MHz}$ applications. Also, an RFID antenna has been designed for ultrahigh frequency (UHF) at 860 $960 \mathrm{MHz}$, which has been verified by both simulation and experiments. The results demonstrated that the UHF RFID antenna can provide a read range of $3 \mathrm{~m}-4 \mathrm{~m}$ with a minimum threshold power of $23-26 \mathrm{dBm}$. Furthermore, a dielectric resonator antenna (DRA) array has been presented herein for giving a good service for GNSS to exploit the potentialities of precise and reliable navigation. The antenna operates at E5/L5, L2, and E6 bands with an overall footprint of only $3.5^{\prime \prime}$. In addition, an optimal electric dipole antennas model has been presented, analyzed, and measured in a through-the-earth communication system to obtain a vertical transmission depth about $30 \mathrm{~m}$.

We hope that this special issue can give useful information and light the antenna designs within the minimization and high performance and can attract much more attention by the academia and industrial community to pursue further investigations to promote the rapid design of small antennas. 


\section{Acknowledgments}

We appreciatingly thank all the authors for their useful designs and informative contributions. We also give thanks to all the reviewers for their continuous support and their constructive comments for promoting the publication process.

Wenhua Yu

Yingsong $L i$

Manos M. Tentzeris 


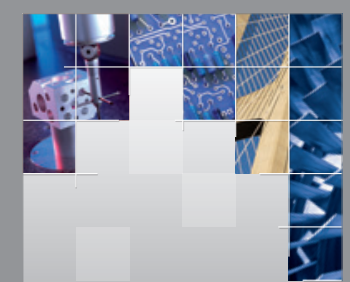

\section{Enfincering}
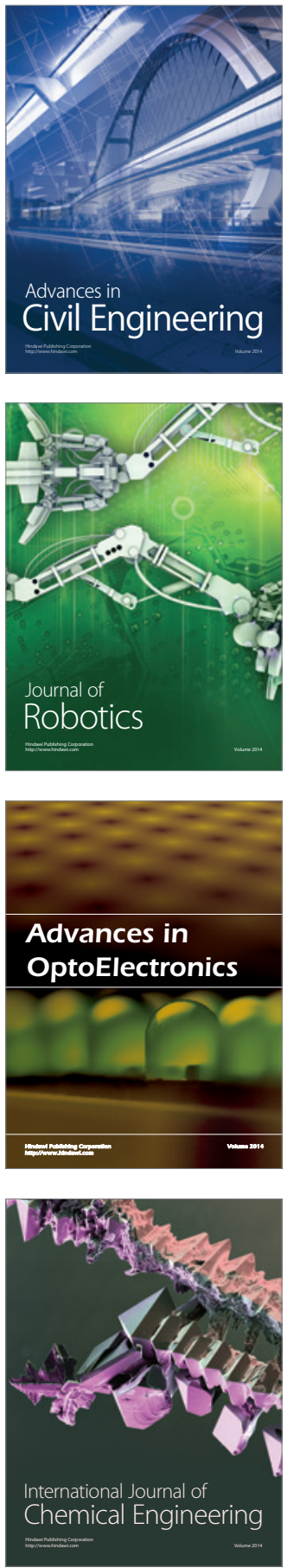

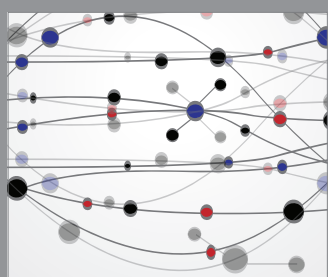

The Scientific World Journal

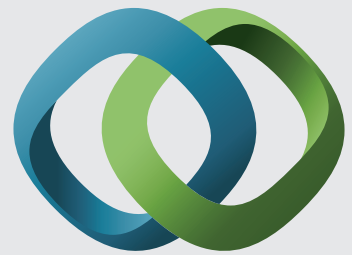

\section{Hindawi}

Submit your manuscripts at

http://www.hindawi.com
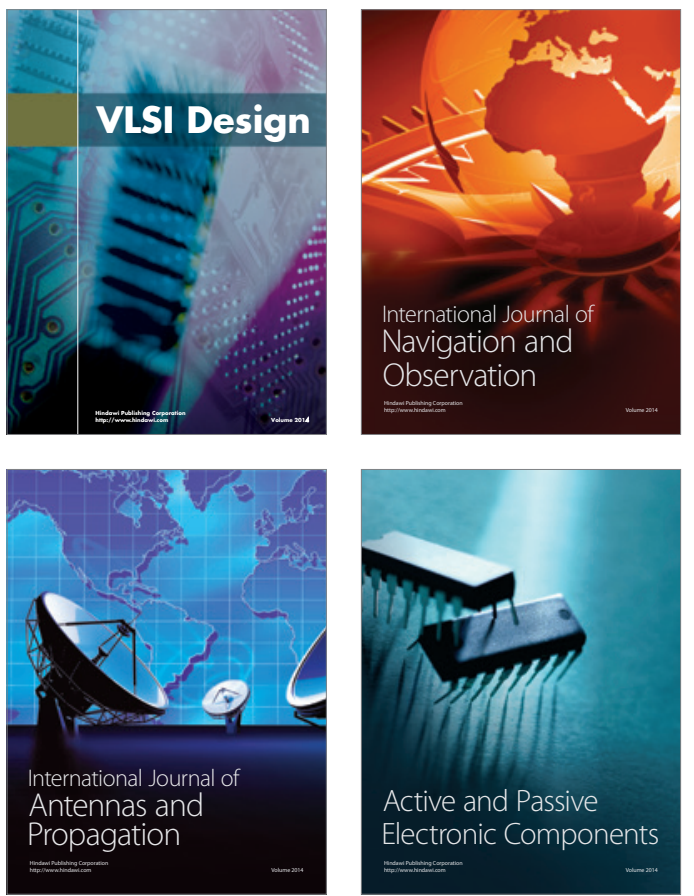
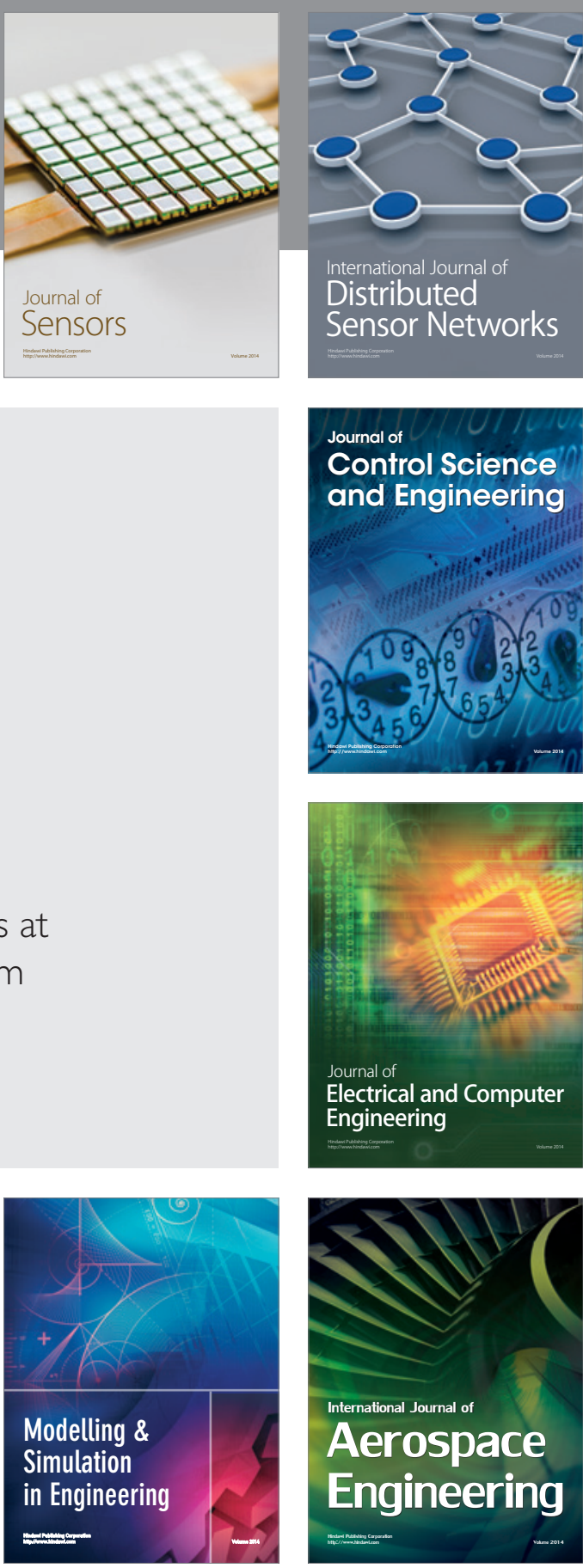

International Journal of

Distributed

Sensor Networks

Journal of

Control Science

and Engineering
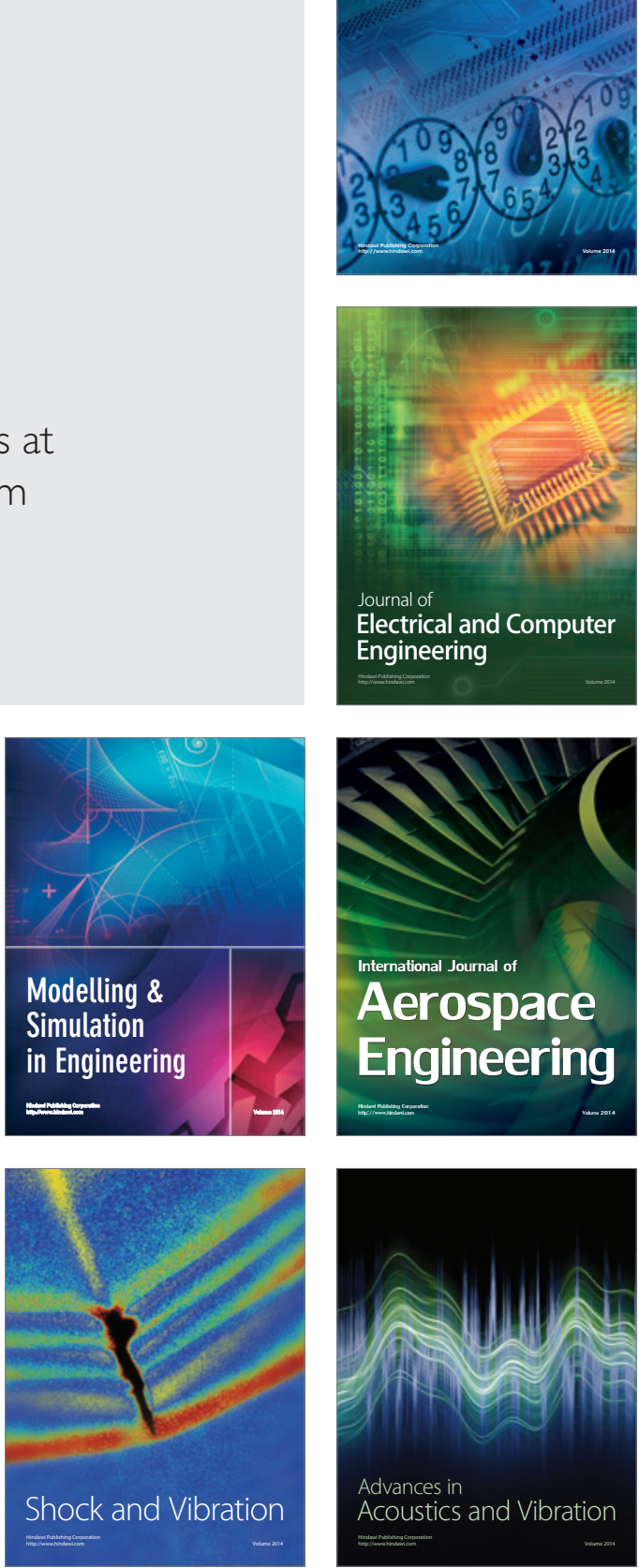